\title{
Two-setting Bell Inequalities for Graph States
}

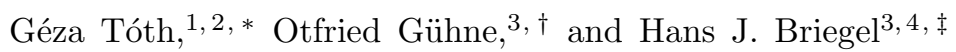 \\ ${ }^{1}$ Max-Planck-Institut für Quantenoptik, Hans-Kopfermann-Straße 1, D-85748 Garching, Germany, \\ ${ }^{2}$ Research Institute of Solid State Physics and Optics, \\ Hungarian Academy of Sciences, H-1525 Budapest P.O. Box 49, Hungary \\ ${ }^{3}$ Institut für Quantenoptik und Quanteninformation, \\ Österreichische Akademie der Wissenschaften, A-6020 Innsbruck, Austria, \\ ${ }^{4}$ Institut für Theoretische Physik, Universität Innsbruck, Technikerstraße 25, A-6020 Innsbruck, Austria
}

(Dated: June 19, 2018)

\begin{abstract}
We present Bell inequalities for graph states with a high violation of local realism. In particular, we show that there is a basic Bell inequality for every nontrivial graph state which is violated by the state at least by a factor of two. This inequality needs the measurement of at most two operators for each qubit and involves only some of the qubits. We also show that for some families of graph states composite Bell inequalities can be constructed such that the violation of local realism increases exponentially with the number of qubits. We prove that some of our inequalities are facets of the convex polytope containing the many-body correlations consistent with local hidden variable models. Our Bell inequalities are built from stabilizing operators of graph states.

PACS numbers: 03.65.Ud, 03.67.-a, 03.67.Lx, 03.67.Pp
\end{abstract}

\section{INTRODUCTION}

Bell inequalities [1, 2, 3, 4, 5, 6, 7, 8] have already been used for several decades as an essential tool for pointing out the impossibility of local realism in describing the results arising from correlation measurements on quantum states. While the relatively young theory of quantum entanglement [9] is also used to characterize the nonclassical behavior of quantum systems, Bell inequalities still remain essential both from the fundamental point of view and also from the point of view of quantum information processing applications. For example, the violation of a two-setting Bell inequality indicates that there is a partition of the multi-qubit quantum state to two parties such that some pure entanglement can be distilled [10]. Furthermore, any state which violates a Bell inequality can be used for reducing the communication complexity of certain tasks [11].

This paper is devoted to the study of the non-local properties of graph states. Graph states 12, 13, 14, 15] are a family of multi-qubit states which comprises many useful quantum states such as the GreenbergerHorne-Zeilinger (GHZ, 16]) states and the cluster states 17. They play an important role in applications: Measurement-based quantum computation uses graph states as resources 18, 19] and all codewords in the standard quantum error correcting codes correspond to graph states [20].

From a theoretical point of view one remarkable fact about graph states is that they can elegantly be described in terms of their stabilizing operators. This means that

\footnotetext{
*Electronic address: toth@alumni.nd.edu

${ }^{\dagger}$ Electronic address: otfried.guehne@uibk.ac.at

‡Electronic address: hans.briegel@uibk.ac.at
}

a graph state can be defined as an eigenstate of several such locally measurable observables. These observables form a commutative group called stabilizer [21]. Stabilizer theory has already been used to study the nonlocal properties of special instances of graph states [22, 23]. In a previous work we showed that for every non-trivial graph state it is possible to construct three-setting Bell inequalities which are maximally violated only by this state [24]. These inequalities use all the elements of the stabilizer.

In this paper we will examine how to create efficient two-setting Bell inequalities for graph states by using only some of the elements of the stabilizer. Efficiency in this case means that our inequalities allow for a high violation of local realism. Apart from trivial graphs, this is at least a factor of two and increases exponentially with the size for some families of graph states. Interestingly, our inequalities are Mermin- and Ardehali-type inequalities with multi-qubit observables [3, 4]. Our Mermintype inequalities are based on a GHZ-type violation of local realism [22]. This means that all correlation terms in our Bell inequalities are +1 for a given graph state, while there is not a local hidden variable (LHV) model with such correlations.

Our paper is organized as follows. In Sec. II we recall the basic facts about graph states and the stabilizer formalism. We also explain the notation we use to formulate our Bell inequalities and provide a first example for a Bell inequality for graph states. In Sec. III we present Bell inequalities for general graphs involving the stabilizing operators of a vertex and its neighbors. These are Mermin-type inequalities with multi-qubit observables. Then, in Sec. IV we discuss how to construct inequalities having a violation of local realism increasing exponentially with the number of qubits for some families of graph states. In Sec. V we present Ardehali-type inequalities with multi-qubit observables which have a higher vi- 
(a)
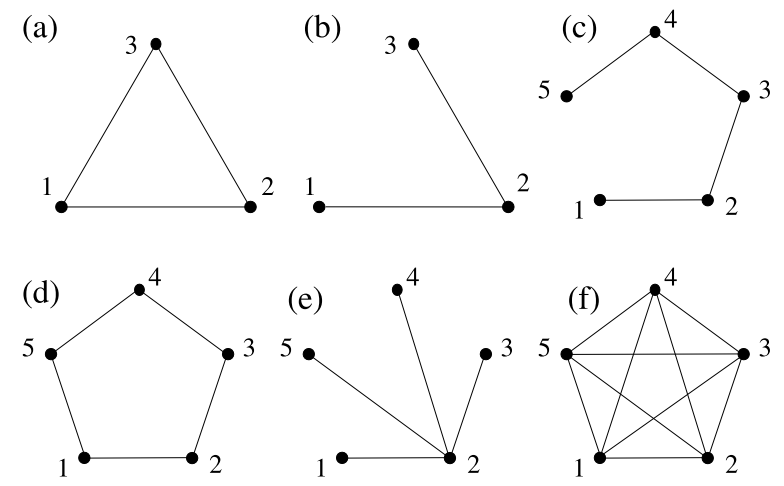

FIG. 1: Examples of graphs: (a) The three-vertex fully connected graph $F C_{3}$. (b) The three-vertex linear cluster graph $L C_{3}$. (c) The five-vertex linear cluster graph $L C_{5}$. (d) The ring cluster graph $R C_{5}$. (e) The star graph $S C_{5}$. (f) The fully connected graph $F_{3}$ with five vertices. Examples of more complicated graphs are shown in Fig. 2.

olation in some cases than the Mermin-type inequalities. In Sec. VI we will show that some of our inequalities correspond to facets (maximal faces) of the convex polytope containing correlations allowed by LHV models. Finally, in Sec. VII we will discuss the connection of our inequalities to existing inequalities for four-qubit cluster states.

\section{DEFINITIONS AND NOTATIONS}

Let us start by briefly recalling the definition of graph states. A detailed investigation of graph states can be found in Ref. [13]. A graph state corresponds to a graph $G$ consisting of $n$ vertices and some edges. Some simple graphs are shown in Fig. 1. Let us characterize the connectivity of this graph by $\mathcal{N}(i)$, which gives the set of neighbors for vertex $i$. We can now define for each vertex a locally measurable observable via

$$
g_{k}:=X^{(k)} \prod_{l \in \mathcal{N}(k)} Z^{(l)},
$$

where $X^{(k)}$ and $Z^{(k)}$ are Pauli spin matrices. A graph state $\left|G_{n}\right\rangle$ of $n$ qubits is now defined to be the state which has these $g_{k}$ as stabilizing operators. This means that the $g_{k}$ have the state $\left|G_{n}\right\rangle$ as an eigenstate with eigenvalue +1 ,

$$
g_{k}\left|G_{n}\right\rangle=\left|G_{n}\right\rangle
$$

In fact, not only the $g_{k}$, but also their products (for example $g_{1} g_{2}, g_{1} g_{3} g_{4}$, etc.) stabilize the state $\left|G_{n}\right\rangle$. These $2^{n}$ operators will be denoted by $\left\{S_{m}\right\}_{m=1}^{2^{n}}$. They form a commutative group called the stabilizer and $\left\{g_{k}\right\}_{k=1}^{n}$ are the generators of this group [21]. All the elements $S_{m}$ of the stabilizer are products of Pauli spin matrices.

Let us fix the notation for formulating Bell inequalities. A Bell operator is typically presented as the sum of many-body correlation terms. Now we will consider Bell operators $\mathcal{B}$ which are the sum of some of the stabilizing operators

$$
\mathcal{B}=\sum_{m \in J} S_{m}
$$

where set $J$ tells us which stabilizing operators we use for $\mathcal{B}$. Since all $S_{m}$ are the products of Pauli spin matrices $X^{(k)}, Y^{(k)}$ and $Z^{(k)}$, we naturally assume that for our Bell inequalities for each qubit these spin coordinates are measured.

The maximum of the mean value $\langle\mathcal{B}\rangle$ for quantum states can immediately be obtained: For the graph state $\left|G_{n}\right\rangle$ all stabilizing operators have an expectation value +1 thus $\langle\mathcal{B}\rangle$ is an integer and it equals the number of stabilizing operators used for constructing $\mathcal{B}$ as given in Eq. (3). Clearly, there is no quantum state for which $\langle\mathcal{B}\rangle$ could be larger.

Now we will determine the maximum of $\langle\mathcal{B}\rangle$ for LHV models. It can be obtained in the following way: We take the definition Eq. (33). We then replace the Pauli spin matrices with real (classical) variables $X_{k}, Y_{k}$ and $Z_{k}$. Let us denote the expression obtained this way by $\mathcal{E}(\mathcal{B})$ :

$$
\mathcal{E}(\mathcal{B}):=\left.[\mathcal{B}]\right|_{X^{(k)} \rightarrow X_{k}, Y^{(k)} \rightarrow Y_{k}, Z^{(k)} \rightarrow Z_{k}} .
$$

It is known that when maximizing $\mathcal{E}(\mathcal{B})$ for LHV models it is enough to consider deterministic LHV models which assign a definite +1 or -1 to the variables $X_{k}, Y_{k}$ and $Z_{k}$. The value of our Bell operators for a given deterministic local model $\mathcal{L}$, i.e., an assignment of +1 or -1 to the classical variables will be denoted by $\mathcal{E}_{\mathcal{L}}(\mathcal{B})$. Thus we can obtain the maximum of the absolute value of $\langle\mathcal{B}\rangle$ for LHV models as

$$
\mathcal{C}(\mathcal{B}):=\max _{\mathcal{L}}\left|\mathcal{E}_{\mathcal{L}}(\mathcal{B})\right|
$$

The usefulness of a Bell inequality in experiments can be characterized by the ratio of the quantum and the classical maximum

$$
\mathcal{V}(\mathcal{B}):=\frac{\max _{\Psi}\left|\langle\mathcal{B}\rangle_{\Psi}\right|}{\max _{\mathcal{L}}\left|\mathcal{E}_{\mathcal{L}}(\mathcal{B})\right|}
$$

Thus $\mathcal{V}(\mathcal{B})$ is the maximal violation of local realism allowed by the Bell operator $\mathcal{B}$. For LHV models we have $\langle\mathcal{B}\rangle \leq \mathcal{C}(\mathcal{B})$. If $\mathcal{V}(\mathcal{B})>1$ then this inequality is a Bell inequality, and some quantum states violate it. In general, the larger $\mathcal{V}(\mathcal{B})$ is, the better our Bell inequality is.

As a warming up exercise, let us now write down explicitly a Bell inequality for a three-qubit linear cluster state $\left|L C_{3}\right\rangle$ [see Fig. 1(b)]. We define

$$
\begin{aligned}
\mathcal{B}^{\left(L C_{3}\right)} & :=Z^{(1)} X^{(2)} Z^{(3)}+Y^{(1)} Y^{(2)} Z^{(3)}+ \\
& +Z^{(1)} Y^{(2)} Y^{(3)}-Y^{(1)} X^{(2)} Y^{(3)}
\end{aligned}
$$

Here $\mathcal{B}^{\left(L C_{3}\right)}$ is given as the sum of four stabilizing operators of $\left|L C_{3}\right\rangle$. For $\left|L C_{3}\right\rangle$ all of these terms have an 
expectation value +1 and the maximum of $\left\langle\mathcal{B}^{\left(L C_{3}\right)}\right\rangle$ for quantum states is 4 . For classical variables, we have

$$
Z_{1} X_{2} Z_{3}+Y_{1} Y_{2} Z_{3}+Z_{1} Y_{2} Y_{3}-Y_{1} X_{2} Y_{3} \leq 2
$$

thus we have $\mathcal{C}\left(\mathcal{B}^{\left(L C_{3}\right)}\right)=2$. The maximal violation of local realism is $\mathcal{V}\left(\mathcal{B}^{\left(L C_{3}\right)}\right)=2$. The inequality Eq. (8), apart from relabeling the variables, has been presented by Mermin 3] for GHZ states. This is not surprising, since the state $\left|L C_{3}\right\rangle$ is, up to local unitary transformations, the GHZ state [13]. It is instructive to write down the Bell operator of Eq. (8) with the $g_{k}^{\left(L C_{3}\right)}$ operators of the three-qubit linear cluster state

$$
\mathcal{B}^{\left(L C_{3}\right)}=g_{2}^{\left(L C_{3}\right)}\left(1+g_{3}^{\left(L C_{3}\right)}\right)\left(1+g_{1}^{\left(L C_{3}\right)}\right),
$$

where, based on Eq. (1), we have $g_{1}^{\left(L C_{3}\right)}=X^{(1)} Z^{(2)}$, $g_{2}^{\left(L C_{3}\right)}=Z^{(1)} X^{(2)} Z^{(3)}$ and $g_{3}^{\left(L C_{3}\right)}=Z^{(2)} X^{(3)}$. Now the question is what happens if new vertices are added to our three-vertex linear graph and spins 1, 2 and 3 have new neighbors as shown in Fig. 22(a). For this case the Bell operator of the form Eq. (9) will be generalized in the following.

Finally, before starting our main discussion, let us recall one important fact which simplifies the calculation of the maximum mean value for local realistic models:

Lemma 1. Let $\mathcal{B}$ be a Bell operator consisting of a subset of the stabilizer for some graph state. Then, when computing the classical maximum $\mathcal{C}(\mathcal{B})$ one can restrict the attention to LHV models which assign +1 to all $Z_{k}$. Proof. The proof of this fact was given in Ref. 24], we repeat it here for completeness. From the construction of graph states and the multiplication rules for Pauli matrices it is easy to see that for an element $S$ of the stabilizer the following fact holds: we have $Y^{(i)}, Z^{(i)}$ at the qubit $i$ in $S$ iff the number of $Y^{(k)}$ and $X^{(k)}$ in the neighborhood $\mathcal{N}(i)$ in $S$ is odd. Thus, if a LHV model assigns -1 to $Z^{(i)}$, we can, by changing the signs for $Z^{(i)}, Y^{(i)}$ and for all $X^{(k)}$ and all $Y^{(k)}$ with $k \in \mathcal{N}(i)$, obtain a LHV model with the same mean value of $\mathcal{B}$ and the desired property.

\section{BELL INEQUALITY ASSOCIATED WITH A VERTEX AND ITS NEIGHBORHOOD}

Now we describe a method which assigns a Bell inequality to each vertex in the graph. The inequality is constructed such that it is maximally violated by the state $|G\rangle$, having stabilizing operators $g_{k}$.

Theorem 1. Let $i$ be a vertex and let $I \subseteq \mathcal{N}(i)$ be a subset of its neighborhood, such that none of the vertices in $I$ are connected by an edge. Then the following operator

$$
\mathcal{B}(i, I):=g_{i} \prod_{j \in I}\left(1+g_{j}\right)
$$

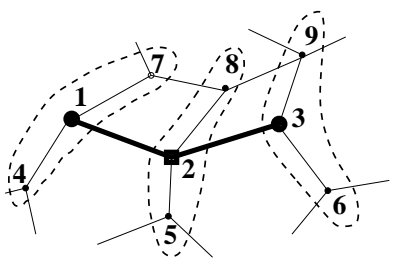

(a)

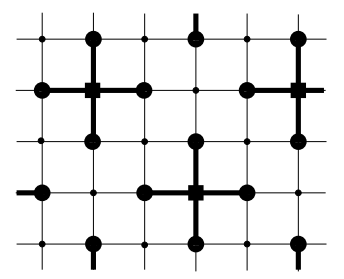

(c)

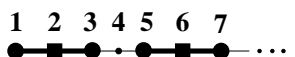

(b)

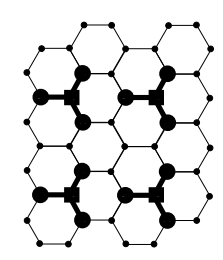

(d)
FIG. 2: Bell inequalities for graph states. (a) The graphical representation of a Bell inequality involving the generators of vertices 1, 2 and 3 . The corresponding three-vertex subgraph is shown in bold. The dashed lines indicate the three qubit-groups involved in this inequality. For the interpretation of symbols at the vertices see text. (b) Graphical representation for Bell inequalities for linear cluster states, (c) a two-dimensional lattice and (d) a hexagonal lattice.

defines a Bell inequality $|\langle\mathcal{B}(i, I)\rangle| \leq L_{M}(|I|+1)$ with

$$
L_{M}(m):= \begin{cases}2^{\frac{m-1}{2}} & \text { for odd } m, \\ 2^{\frac{m}{2}} & \text { for even } m\end{cases}
$$

and $|G\rangle$ maximally violates it with $\langle\mathcal{B}(i, I)\rangle=2^{|I|}$. The notation $\mathcal{B}(i, I)$ indicates that the Bell operator for our inequality is constructed with the generators corresponding to vertex $i$ and to some of its neighbors given by set I.

Proof. Let us first consider a concrete example shown in Fig. 2(a). Consider vertex 2 and its two neighbors, vertices 1 and 3 . Now constructing the Bell operator $\mathcal{B}$ involves $g_{2}$ and $\left(1+g_{1 / 3}\right)$. This is expressed by denoting these vertices by squares and disks, respectively. The three-vertex subgraph with bold edges now represents the Bell inequality $\mathcal{B}(2,\{1,3\})$. Then by expanding the brackets in Eq. (10) one obtains

$$
\begin{aligned}
\mathcal{B}(2,\{1,3\})=Z^{(1)}\left(Z^{(5)} Z^{(8)} X^{(2)}\right) Z^{(3)} \\
\quad+\left(Z^{(4)} Z^{(7)} Y^{(1)}\right)\left(Z^{(5)} Z^{(8)} Y^{(2)}\right) Z^{(3)} \\
\quad+Z^{(1)}\left(Z^{(5)} Z^{(8)} Y^{(2)}\right)\left(Z^{(6)} Z^{(9)} Y^{(3)}\right) \\
\quad-\left(Z^{(4)} Z^{(7)} Y^{(1)}\right)\left(Z^{(5)} Z^{(8)} X^{(2)}\right)\left(Z^{(6)} Z^{(9)} Y^{(3)}\right) .
\end{aligned}
$$

Eq. (12) corresponds to measuring two multi-spin observables at each of three parties, where a party is formed out of several qubits. In fact, in Eq. (12) one can recognize the three-body Mermin inequality with multi-qubit 
observables. These observables are indicated by bracketing. The corresponding three qubit groups are indicated by dashed lines in Fig. 2(a). Note that if vertices 1 and 3 were connected by an edge then an operator of the form Eq. (10) would involve three different variables at sites 1 and 3 .

Let us now turn to the general case of an inequality involving a vertex and its $N_{\text {neigh }} \geq 2$ neighbors given in $\left\{I_{k}\right\}_{k=1}^{N_{\text {neigh }}}$. Then, similarly to the previous tripartite example, this inequality is effectively a $(|I|+1)$-body Mermin's inequality. In order to see that, let us define the reduced neighborhood of vertex $k$ as

$$
\tilde{\mathcal{N}}(k):=\mathcal{N}(k) \backslash(I \cup\{i\}) .
$$

Then we define the following multi-qubit observables

$$
\begin{aligned}
A^{(1)} & :=Y^{(i)} \prod_{k \in \tilde{\mathcal{N}}(i)} Z^{(k)}, \\
B^{(1)} & :=X^{(i)} \prod_{k \in \tilde{\mathcal{N}}(i)} Z^{(k)}, \\
A^{(j+1)} & :=Z^{\left(I_{j}\right)}, \\
B^{(j+1)} & :=Y^{\left(I_{j}\right)}\left(\prod_{k \in \tilde{\mathcal{N}}\left(I_{j}\right)} Z^{(k)}\right),
\end{aligned}
$$

for $j=1,2, \ldots, N_{\text {neigh }}$ and $I_{j}$ denotes the $j$-th element of $I$. Then we can write down our Bell operator given in Eq. (10) as the Bell operator of a Mermin inequality with $A^{(i)}$ and $B^{(i)}$

$$
\begin{aligned}
\mathcal{B}(i, I) & =\sum_{\pi} B^{(1)} A^{(2)} A^{(3)} A^{(4)} A^{(5)} \ldots \\
& -\sum_{\pi} B^{(1)} B^{(2)} B^{(3)} A^{(4)} A^{(5)} \ldots \\
& +\sum_{\pi} B^{(1)} B^{(2)} B^{(3)} B^{(4)} B^{(5)} \cdots
\end{aligned}
$$

where $\sum_{\pi}$ represents the sum of all possible permutations of the qubits that give distinct terms. Hence the bound for local realism for Eq. (10) is the same as for the $(|I|+$ 1)-partite Mermin inequality [3, 25]. For $|G\rangle$ all the terms in the Mermin inequality using the variables defined in Eq. (14) have an expectation value +1 thus $\langle\mathcal{B}(i, I)\rangle=$ $2^{|I|}$.

There is also an alternative way to understand why the extra $Z^{(k)}$ terms in the Bell operator does not influence the maximum for LHV models. I.e., the maximum is the same as for the $(|I|+1)$-qubit Mermin inequality. For that we have to use Lemma 1 described for computing the maximum for LHV models for an expression constructed as a sum of stabilizer elements of a graph state. Lemma 1 says that the $Z_{k}$ terms can simply be set to +1 and for computing the maximum it is enough to vary the $X_{k}$ and $Y_{k}$ terms. Thus from the point of view of the maximum, the extra $Z_{k}$ terms can be neglected and would not change the maximum for LHV models even if it were not possible to reduce our inequality to a $(|I|+1)$ body Mermin inequality using the definitions Eq. (14).

Furthermore, it is worth noting that the above presented inequalities can be viewed as conditional Mermin inequalities for qubits $\{i\} \cup I$ after $Z^{(j)}$ measurements on the neighboring qubits are performed [26]. Indeed, after measuring $Z^{(j)}$ on these qubits, a state locally equivalent to a GHZ state is obtained. Knowing the outcomes of the $Z^{(j)}$ measurements, one can determine which state it is exactly and can write down a Mermin-type inequality with two single-qubit measurements per site which is maximally violated by this state. Indeed, this Mermintype inequality can be obtained from the Bell inequality presented in Eqs. (10 11) in Theorem 1, after substituting in it the \pm 1 measurement results for these $Z^{(j)}$ measurements. Our scheme shows some relation to the Bell inequalities presented in Ref. [5]. These were essentially two-qubit Bell inequalities conditioned on measurement results on the remaining qubits.

Finally, we can state:

Theorem 2. Every nontrivial graph state violates a twosetting Bell inequality at least by a factor of two.

Proof. Every nontrivial graph state has at least one vertex $i$ with at least two neighbors, $j$ and $k$. There are now two possibilities: (i) If these two neighbors are not connected to each other by an edge then Theorem 1 provides a Bell inequality with a Bell operator $\mathcal{B}(i,\{j, k\})$ which is violated by local realism by a factor of two. (ii) If these two neighbors are connected by an edge, then the situation of Fig.1(a) occurs. In this case, we look at the Bell operator

$\mathcal{B}^{\left(F C_{3}\right)}:=g_{1}^{\left(F C_{3}\right)}+g_{2}^{\left(F C_{3}\right)}+g_{3}^{\left(F C_{3}\right)}+g_{1}^{\left(F C_{3}\right)} g_{2}^{\left(F C_{3}\right)} g_{3}^{\left(F C_{3}\right)}$.

As before, one can now show that this results in a Bell inequality which is equivalent to the three-qubit Mermin inequality.

\section{COMPOSITE BELL INEQUALITIES}

Theorem 1 can also be used to obtain families of Bell inequalities with a degree of violation increasing exponentially with the number of qubits. In order to do this, let us start from two Bell inequalities of the form

$$
\begin{gathered}
\left|\mathcal{E}_{1}\right| \leq \mathcal{C}_{1}, \\
\left|\mathcal{E}_{2}\right| \leq \mathcal{C}_{2} .
\end{gathered}
$$

where $\mathcal{E}_{1 / 2}$ denote two expressions with classical variables $X_{k}$ 's, $Y_{k}$ 's and $Z_{k}$ 's. Then it follows immediately that

$$
\left|\mathcal{E}_{1} \mathcal{E}_{2}\right| \leq \mathcal{C}_{1} \mathcal{C}_{2}
$$

Concerning Bell inequalities, one has to be careful at this point: Eq. (18) is not necessarily a Bell inequality. It may happen that $\mathcal{E}_{1} \mathcal{E}_{2}$ have correlation terms which contain two or more variables of the same qubit, e.,g., 
$\left(X_{1} Z_{2}\right)\left(Z_{1} X_{2}\right)=X_{1} Z_{1} X_{2} Z_{2}$. Such a correlation term cannot appear in a Bell inequality. Because of that we need the following theorem.

Theorem 3. Let us consider two Bell inequalities of the form Eq. (17). If for each qubit $k$ at most only one of the inequalities contain variables corresponding to the qubit, then Eq. (18) describes a composite Bell inequality. If both of the inequalities contain variables corresponding to qubit $k$, then Eq. (18) still describes a Bell inequality if the inequalities contain the same variable for qubit $k$. Proof. After the previous discussion it is clear that none of the correlation terms of the Bell inequality Eq. (18) contain more than two variables for a qubit. They may contain quadratic terms such as $X_{k}^{2}$, however, these can be replaced by 1 .

Let us now consider two of our Bell inequalities, $\mathcal{B}\left(i, I_{i}\right)$ and $\mathcal{B}\left(j, I_{j}\right)$, where $I_{i / j} \subset \mathcal{N}(i / j)$. From now let us omit the second index after $\mathcal{B}$. Then based on the previous ideas a new composite Bell inequality can be constructed with a Bell operator $\mathcal{B}:=\mathcal{B}(i) \mathcal{B}(j)$ if the qubits in $\{i\} \cup I_{i}$ and the qubits in $\{j\} \cup I_{j}$ are not neighbors [27]. For the composite inequality $\mathcal{C}(\mathcal{B})=\mathcal{C}[\mathcal{B}(i)] \mathcal{C}[\mathcal{B}(j)]$ and $\mathcal{V}(\mathcal{B})=$ $\mathcal{V}[\mathcal{B}(i)] \mathcal{V}[\mathcal{B}(j)]$ thus the violation of local realism is larger for the composite inequality than for the two original inequalities.

Based on these ideas, composite Bell inequalities can be created from several inequalities. Let us see a concrete example. For an $n$-qubit cluster state we have the stabilizing operators $g_{i}^{\left(L C_{n}\right)}:=Z^{(i-1)} X^{(i)} Z^{(i+1)}$ where $i \in\{1,2, . ., n\}$ and for the boundaries $Z^{(0)}=Z^{(n+1)}=\mathbb{1}$. Then we can define the following Bell inequality for vertex $i$

$$
\begin{aligned}
\mathcal{B}_{i}^{\left(L C_{n}\right)} & :=g_{i}^{\left(L C_{n}\right)}\left(1+g_{i+1}^{\left(L C_{n}\right)}\right)\left(1+g_{i-1}^{\left(L C_{n}\right)}\right) \\
& =Z^{(i-1)} X^{(i)} Z^{(i+1)}+Z^{(i-2)} Y^{(i-1)} Y^{(i)} Z^{(i+1)} \\
& +Z^{(i-1)} Y^{(i)} Y^{(i+1)} Z^{(i+2)} \\
& -Z^{(i-2)} Y^{(i-1)} X^{(i)} Y^{(i+1)} Z^{(i+2)} \\
\mathcal{V}\left(\mathcal{B}_{i}^{\left(L C_{n}\right)}\right) & =2 .
\end{aligned}
$$

Now we can combine these Bell inequalities, for different $i$ as illustrated in Fig. 2(b). Here $\mathcal{B}_{2}^{\left(L C_{n}\right)}$ and $\mathcal{B}_{6}^{\left(L C_{n}\right)}$ are represented by two bold subgraphs. If $n$ is divisible by four then we obtain a composite inequality characterized by

$$
\begin{aligned}
\mathcal{B}^{\left(L C_{n}\right)} & :=\prod_{i=1}^{n / 4} \mathcal{B}_{4 i-2}^{\left(L C_{n}\right)}, \\
\mathcal{V}\left(\mathcal{B}^{\left(L C_{n}\right)}\right) & =2^{n / 4} .
\end{aligned}
$$

Thus the violation increases exponentially with $n$.
These ideas can be generalized for a two-dimensional lattice as shown in Fig. 2(c). Here 5-body Bell inequalities, represented again by bold subgraphs in the figure, can be combined in order to obtain a violation of lo-

\begin{tabular}{|c|c|c|c|c|c|c|c|c|c|c|}
\hline Number of qubits & $n$ & 34 & & 6 & & & 9 & 10 & 11 & 12 \\
\hline Linear cluster graph & $L C_{n}$ & 22 & & 2 & & & 4 & 4 & 8 & 8 \\
\hline Ring cluster graph & $R C_{n}$ & 22 & & 2 & & & 4 & 4 & 4 & 8 \\
\hline Star graph & $S T_{n}$ & \begin{tabular}{|l|l|l}
2 & 2 \\
\end{tabular} & 2 & 4 & & 8 & 16 & 16 & 32 & 32 \\
\hline
\end{tabular}
cal realism increasing exponentially with the number of vertices. Bell inequalities are also shown this way for a

TABLE I: Maximal violation $\mathcal{V}$ of local realism for composite Bell inequalities for various interesting graph states as a function of the number of qubits. These composite inequalities are constructed from the inequalities of Theorem 1.

hexagonal lattice in Fig. 2(d). These ideas can straightforwardly be generalized for arbitrary graphs.

In Table I the relative violation of local realism is shown for some interesting graph states as the function of the number of qubits for Bell inequalities constructed based on the previous ideas. The state corresponding to a star graph is equivalent to a GHZ state. The corresponding inequality is equivalent to Mermin's inequality under relabeling the variables and it has the highest violation of local realism for a given number of qubits.

\section{ALTERNATIVE BELL INEQUALITIES WITH MULTI-QUBIT VARIABLES}

In Theorem 1 we presented Mermin-type Bell inequalities with multi-qubit observables for graph states. Now we will show that Ardehali-type inequalities can also be constructed and these have a higher violation of local realism for odd $|I|$. Note that they are not constructed from stabilizing terms.

Theorem 4. Let us consider vertex $i$ which has $N_{\text {neigh }} \geq$ 2 neighbors given in $I=\left\{I_{k}\right\}_{k=1}^{N_{\text {neigh }}}$ such that they are not connected by edges. Let furthermore $A^{(k)}$ and $B^{(k)}$ be defined as in Eq. (14) and define also

$$
\begin{aligned}
Q^{(1)} & :=\frac{A^{(1)}-B^{(1)}}{\sqrt{2}}, \\
W^{(1)} & :=\frac{A^{(1)}+B^{(1)}}{\sqrt{2}} .
\end{aligned}
$$

Then, we can write down the following Bell inequality 


$$
\begin{aligned}
& \left(Q_{1}-W_{1}\right)\left(-\sum_{\pi} A_{2} A_{3} A_{4} A_{5} \cdots A_{M}+\sum_{\pi} B_{2} B_{3} A_{4} A_{5} \cdots A_{M}-\sum_{\pi} B_{2} B_{3} B_{4} B_{5} \cdots A_{M} \cdots\right) \\
+ & \left(Q_{1}+W_{1}\right)\left(\sum_{\pi} B_{2} A_{3} A_{4} A_{5} \cdot A_{M}-\sum_{\pi} B_{2} B_{3} B_{4} A_{5} \cdots A_{M}+\sum_{\pi} B_{2} B_{3} B_{4} B_{5} \cdot A_{M} \cdots\right) \leq L_{A}(|I|+1),
\end{aligned}
$$

where the bound for the Bell inequality is

$$
L_{A}(m):= \begin{cases}2^{\frac{m+1}{2}} & \text { for odd } m \\ 2^{\frac{m}{2}} & \text { for even } m\end{cases}
$$

Again, $\sum_{\pi}$ represents the sum of all possible permutations of the qubits that give distinct terms. If $A_{k}, B_{k}$, $Q_{1}$ and $W_{1}$ correspond to the measurement of quantum operators $A^{(k)}, B^{(k)}, Q^{(1)}$ and $W^{(1)}$, respectively, then the graph state $|G\rangle$ maximally violates Eq. (22). Note that similarly as in Theorem 1 a party consists of several qubits here.

Proof. The bound for LHV models is valid since Eq. (22) is the Bell operator of the $(|I|+1)$-body Ardehali's Bell inequality [4, 28] for $Q_{1}, W_{1}$, and the $A_{k}$ 's and $B_{k}$ 's. For the same reason, the maximal value for the Bell operator (22) for quantum states is the same as for Ardehali's inequality, i.e., $2^{|I|} \sqrt{2}$. This value is obtained for $|G\rangle$ as we will show. In order to see that let us substitute the definitions of $Q^{(1)} / W^{(1)}$ given in Eq. (21) into the Bell operator of the inequality Eq. (22). That is, let us substitute $-\sqrt{2} B^{(1)}$ for $\left(Q^{(1)}-W^{(1)}\right)$ and $\sqrt{2} A^{(1)}$ for $\left(Q^{(1)}+W^{(1)}\right)$. Then expand the brackets. This way one obtains the Bell operator as the sum of $2^{|I|}$ terms. These terms correspond to stabilizing operators multiplied by $\sqrt{2}$.

\section{PROVING THAT OUR INEQUALITIES ARE EXTREMAL}

Based on Ref. 8], we know that our Mermin-type inequalities given in Theorem 1 have a maximal violation of local realism $\mathcal{V}$ [defined in Eq. [6]) among $(|I|+1)$ partite Bell inequalities for even $|I|$. Here this statement is valid for inequalities analyzed in Ref. 7, 8, which need the measurement of two observables for each party and they are the weighted sum of full correlation terms. The same is true for the Ardehali-type inequalities given in Theorem 4 for odd $|I|$ [29]. Moreover, we can prove the following:

Theorem 5. Our Mermin-type inequalities given in Theorem 1 are extremal for even $|I|$, i.e., they are facets of the convex polytope of correlations consistent with LHV models. This is also true for our Ardehali-type inequalities given in Theorem 4 for odd $|I|$. Combining them one obtains also extremal inequalities.

Proof. If we did not have many-body observables then it would be clear that our Bell inequalities are facets [8]. It has also been proved that by multiplying some of these inequalities with each other, extremal inequalities are obtained [8]. Now, however, we have to prove that by replacing some of the variables by the products of several variables does not change this property [30]. First of all, one has to stress that when drawing the convex polytope, the axes correspond to the expectation values of the many-body correlations terms appearing in the Bell inequality [31]. Clearly, replacing a variable with several variables by inserting $Z^{(k)}$ 's in some of these correlation terms does not change the convex polytope of correlations allowed by local models. The transformed Bell inequalities also correspond to the same hyperplane as before the transformation.

\section{COMPARISON WITH EXISTING BELL INEQUALITIES FOR FOUR-QUBIT CLUSTER STATES}

The systematic study of Bell inequalities for graph states was initiated in an important paper by Scarani, Acín, Schenck, and Aspelmeyer 22]. Moreover, in this paper, a Bell inequality for a four-qubit cluster state was presented which has already been used for detecting the violation of local realism experimentally 32]. The inequality of Ref. [22] is also a Mermin's inequality with composite observables

$$
X_{1} X_{3} Z_{4}+Z_{1} Y_{2} Y_{3} Z_{4}+X_{1} Y_{3} Y_{4}-Z_{1} Y_{2} X_{3} Y_{4} \leq 2 .
$$

It is instructive to write down its Bell operator with the stabilizing operator of a cluster state

$$
\mathcal{B}^{\left(L C_{4}\right)}=g_{3}^{\left(L C_{4}\right)}\left(g_{1}^{\left(L C_{4}\right)}+g_{2}^{\left(L C_{4}\right)}\right)\left(\mathbb{1}+g_{4}^{\left(L C_{4}\right)}\right) .
$$

This is different from our ansatz in Eq. (10). The inequality obtained from our ansatz Eq. (10) for $i=3$ is

$$
Z_{2} X_{3} Z_{4}+Z_{1} Y_{2} Y_{3} Z_{4}+Z_{2} Y_{3} Y_{4}-Z_{1} Y_{2} X_{3} Y_{4} \leq 2
$$

The following two four-qubit Bell inequalities are also built with stabilizing terms and have a factor of two violation of local realism:

$$
\begin{array}{r}
X_{1} X_{3} Z_{4}-Y_{1} X_{2} Y_{3} Z_{4}+X_{1} Y_{3} Y_{4}+Y_{1} X_{2} X_{3} Y_{4} \leq 2 \\
Z_{2} X_{3} Z_{4}-Y_{1} X_{2} Y_{3} Z_{4}+Z_{2} Y_{3} Y_{4}+Y_{1} X_{2} X_{3} Y_{4} \leq 2 .
\end{array}
$$


Further four inequalities can be obtained by exchanging qubits 1 and 4, and qubits 2 and 3, in the previous four Bell inequalities. These eight inequalities are all maximally violated by the four-qubit cluster state $\left|L C_{4}\right\rangle$, however, not only by the cluster state. The maximum of the Bell operator for these inequalities is doubly degenerate. Thus, as discussed in Ref. [22] for the case of Eq. (24), they are maximally violated also by some mixed states.

It can be proved by direct calculation that adding any two of these eight inequalities another inequality is obtained such that only the four-qubit cluster state violates it maximally. Thus from the degree of violation of local realism one can also obtain fidelity information, i.e., one can get information on how close the quantum state is to the cluster state. To be more specific, let us see a concrete example for using this fact. Let us denote the Bell operators of the four inequalities in Eqs. 24, 26 27) by $\mathcal{B}_{k}$ with $k=1,2,3,4$. Then direct calculation shows that the following matrix is positive semidefinite

$$
16\left|L C_{4}\right\rangle\left\langle L C_{4}\right|-\mathcal{B}_{1}-\mathcal{B}_{2}-\mathcal{B}_{3}-\mathcal{B}_{4} \geq 0 .
$$

Hence a lower bound on the fidelity can be obtained as

$$
F \geq \frac{1}{16}\left\langle\mathcal{B}_{1}+\mathcal{B}_{2}+\mathcal{B}_{3}+\mathcal{B}_{4}\right\rangle
$$

\section{CONCLUSION}

We discussed how to construct two-setting Bell inequalities for detecting the violation of local realism for quantum states close to graph states. These Bell inequalities allow at least a factor of two violation of local realism. We used the stabilizer theory for constructing our inequalities. For several families of states we have shown that the relative violation increases exponentially with the size. Some of the inequalities presented are facets of the convex polytope corresponding to the correlations permitted by local hidden variable models.

\section{ACKNOWLEDGMENT}

We would like to thank A. Acín, J.I. Cirac, P. Hyllus and C.-Y. Lu for useful discussions. G.T. especially thanks M.M. Wolf for many helpful discussions on Bell inequalities. We also acknowledge the support of the Austrian Science Foundation (FWF), the EU projects RESQ, ProSecCo, OLAQUI, SCALA and QUPRODIS, the DFG and the Kompetenznetzwerk Quanteninformationsverarbeitung der Bayerischen Staatsregierung. G.T. thanks the Marie Curie Fellowship of the European Union (Grant No. MEIF-CT-2003-500183) and the National Research Fund of Hungary OTKA under Contract No. T049234.
[1] J.S. Bell, Physics 1, 195 (1964); for a review see R. Werner and M. Wolf, Quant. Inf. Comp. 1 (3), 1 (2001); for results on multipartite Bell inequalities see Refs. 3, 4, 5, 6, 7, 8 and G. Svetlichny, Phys. Rev. D 35, 3066 (1987); A.V. Belinskii and D.N. Klyshko, Usp. Fiz. Nauk 163 (8), 1 (1993); N. Gisin and H. Bechmann-Pasquinucci, Phys. Lett. A 246, 1 (1998); A. Peres, Found. Phys. 29, 589 (1999); D. Collins, N. Gisin, S. Popescu, D. Roberts, and V. Scarani, Phys. Rev. Lett. 88, 170405 (2002); W. Laskowski, T. Paterek, M. Żukowski, and Č. Brukner, Phys. Rev. Lett. 93, 200401 (2004).

[2] M. Froissart, Nuovo Cimento B 64, 241 (1981).

[3] N.D. Mermin, Phys. Rev. Lett. 65, 1838 (1990).

[4] M. Ardehali, Phys. Rev. A 46, 5375 (1992).

[5] S. Popescu and D. Rohrlich, Phys. Lett. A 166, 293 (1992).

[6] I. Pitowsky and K. Svozil, Phys. Rev. A 64, 014102 (2001).

[7] M. Żukowski and Č. Brukner, Phys. Rev. Lett. 88, 210401 (2002).

[8] R.F. Werner and M.M. Wolf, Phys. Rev. A 64, 032112 (2001).

[9] R.F. Werner, Phys. Rev. A 40, 4277 (1989).
[10] A. Acín, private communication; Phys. Rev. Lett. 88, 027901 (2002); A. Acín, V. Scarani, and M.M. Wolf, Phys. Rev. A 66, 042323 (2002).

[11] Č. Brukner, M. Żukowski, J.-W. Pan, and A. Zeilinger, Phys. Rev. Lett. 92, 127901 (2004).

[12] W. Dür, H. Aschauer, and H.J. Briegel, Phys. Rev. Lett. 91, 107903 (2003).

[13] M. Hein, J. Eisert, and H.J. Briegel, Phys. Rev. A 69, 062311 (2004).

[14] M. Van den Nest, J. Dehaene, and B. De Moor, Phys. Rev. A 72, 014307 (2005); 69, 022316 (2004); 70, 034302 (2004); K.M.R. Audenaert and M.B. Plenio, New J. Phys. 7, 170 (2005); A. Hamma, R. Ionicioiu, and P. Zanardi, Phys. Rev. A 72, 012324 (2005); D.E. Browne and T. Rudolph, Phys. Rev. Lett. 95, 010501 (2005).

[15] For experimental implementations see Refs. [19, 32] and O. Mandel, M. Greiner, A. Widera, T. Rom, T.W. Hänsch and I. Bloch, Nature (London) 425, 937 (2003); A.-N. Zhang, C.-Y. Lu, X.-Q. Zhou, Y.-A. Chen, Z. Zhao, T. Yang, and J.-W. Pan, quant-ph/0501036

[16] D.M. Greenberger, M.A. Horne, A. Shimony, and A. Zeilinger, Am. J. Phys. 58, 1131 (1990).

[17] H.J. Briegel and R. Raussendorf, Phys. Rev. Lett. 86, 910 (2001). 
[18] R. Raussendorf and H.J. Briegel, Phys. Rev. Lett. 86, 5188 (2001); M. Nielsen, ibid., 93, 040503 (2004).

[19] P. Walther, K.J. Resch, T. Rudolph, E. Schenck, H. Weinfurter, V. Vedral, M. Aspelmeyer, and A. Zeilinger, Nature (London) 434, 169 (2005); N. Kiesel, C. Schmid, U. Weber, G. Tóth, O. Gühne, R. Ursin, and H. Weinfurter, Phys. Rev. Lett. 95, 210502 (2005).

[20] D. Schlingemann and R.F. Werner, Phys. Rev. A 65, 012308 (2002); M. Grassl, A. Klappenecker, and M. Rötteler, in Proc. 2002 IEEE International Symposium on Information Theory, Lausanne, Switzerland, p. 45; K. Chen, H.-K. Lo, quant-ph/0404133

[21] D. Gottesman, Phys. Rev. A 54, 1862 (1996).

[22] V. Scarani, A. Acín, E. Schenck, and M. Aspelmeyer, Phys. Rev. A 71, 042325 (2005).

[23] D.P. DiVincenzo and A. Peres, Phys. Rev. A 55, 4089 (1997).

[24] O. Gühne, G. Tóth, P. Hyllus, and H.J. Briegel, Phys. Rev. Lett. 95, 120405 (2005).

[25] If one takes the Bell operator presented in Ref. [3] and replaces $A^{(k)}$ and $B^{(k)}$ by $X^{(k)}$ and $Y^{(k)}$, respectively, then the Bell operator Eq. (15) is obtained.

[26] J.I. Cirac, private communication.
[27] In this case the stabilizing terms in $\mathcal{B}(i)$ and $\mathcal{B}(j)$ commute locally. See G. Tóth and O. Gühne, Phys. Rev. A 72, 022340 (2005).

[28] If one takes the Bell inequality presented in Ref. [4] and interchanges $A_{k} / B_{k}$ with $\sigma_{x / y}^{k-1}$ for $k \geq 2$ and $Q_{1} / W_{1}$ by $\sigma_{a / b}^{n}$, respectively, then inequality Eq. (22) is obtained.

[29] Ref. 8] in Eq.(25) states that the maximal violation of local realism for an $n$-partite Bell inequality with full correlation terms is bounded as $\mathcal{V} \leq 2^{\frac{n-1}{2}}$. Ref. [8] also points out that Mermin inequalities for odd number of parties and Ardehali inequalities for even number of parties give the maximal $\mathcal{V}=2^{\frac{n-1}{2}}$. Note that Ref. [8] uses the term 'the set of inequalities going back to Mermin' in the general sense, denoting the Mermin and Ardehali inequalities giving maximal violation mentioned above.

[30] Stefano Pironio, J. Math. Phys. 46, 062112 (2005).

[31] The coordinate axes could also be the probabilities of the different measurement outcomes rather than the manybody correlation terms. Such an approach was followed, for example, in Ref. [6].

[32] P. Walther, M. Aspelmeyer, K.J. Resch, and A. Zeilinger, Phys. Rev. Lett. 95, 020403 (2005). 